\title{
Comparing Wavelet Transforms and AR Modeling as Feature Extraction Tools for Underwater Signal Classification
}

\author{
Monique P. Fargues \& Richard Bennett \\ Department of Electrical and Computer Engineering \\ Naval Postgraduate School \\ Monterey, CA 93943 \\ fargues@ece.nps.navy.mil
}

\begin{abstract}
This study investigates the application of orthogonal, non-orthogonal Wavelet-based procedures, and AR modeling as feature extraction techniques to classify several classes of underwater signals consisting of sperm whale, killer whale, gray whale, pilot whale, humpback whale, and underwater earthquake data. A two-hidden-layers backpropagation neural network is used for the classification procedure. Performances obtained using the two Wavelet-based schemes are compared with those obtained using reduced-rank AR modeling tools. Results show that the non-orthogonal undecimated A-trous implementation with multiple voices leads to the highest classification rate of $96.7 \%$.
\end{abstract}

Keywords: Wavelets, classification, AR modeling.

\section{Introduction}

The National Oceanic and Atmospheric Administration (NOAA) Pacific Environmental Laboratory conducts on-going studies of underwater geological processes, such as earthquakes and volcanic activities using the Widbey Island undersea SOund Surveillance Systems (SOSUS) array out of Widbey Island, Washington state. It consists of fixed hydrophones mounted at the approximate depth of the deep sound channel, which have the ability to pick up very low frequency sounds. This undersea surveillance system has also great potential for use in tracking and estimating populations of marine mammals as it is located on the migration route of pacific whales. A lot of interest exists in monitoring

The views expressed here are those of the authors and do not reflect the official policy or position of the U.S. Government. such underwater biological sounds in the marine biology community. However, such monitoring requires the study of vast amount of data. Therefore, there is a need for automatic schemes which would detect the presence of specific signals of interest and classify them, thereby significantly reducing the need for human monitoring. The overall goal of the study was twofold; 1) to detect seismic events, 2) to differentiate seismic events from underwater biological sounds and to classify signals in various classes. This paper focuses on the classification aspect only.

\section{Signals Description}

Six different classes of signals were selected for our study: Sperm whale, killer whale, humpback whale, gray whale, pilot whale, and underwater earthquake.

This selection of signals represents a crosssection of biological sounds and seismic events which can be present simultaneously during migration periods along the Pacific North-West coast of the U.S.A. The recordings used in our study were of real, open ocean encounters from various signal collection platforms. The signals, as an artefact of the collection procedures, were all corrupted with background noises, which included sounds from ships, small boats, and other disturbances occurring in the natural environment, plus artificial noise from the means of collection. Each recording varied in length between fifteen to thirty seconds. Each signal was digitized with a sampling frequency equal to $8 \mathrm{kHz}$, using a single channel and 8 bits per sample. Data was then split in non-overlapping blocks of 512 sample points $(64 \mathrm{~ms})$. Four out of five classes of underwater biological signals used in our study were cuts from what is commonly known as "whale songs" 
and were narrowband in nature, while sperm whale and earthquake recordings covered a wider range of frequencies as compared to the first four classes. The sperm whale recordings were of the animal's echo ranging sonar, and constituted of very short and rapid wideband pulses [1]. Figures 1 to 3 presents spectrograms obtained from gray, humpback and sperm whale data.

\section{Wavelet transformations}

Wavelet transforms have numerous applications in signal processing, such as coding, image processing, compression, and classification, and numerous references are available $[1,2]$. In our study we were interested in extracting a compact (i.e., "small") set of feature coefficients which could be used to classify the different signals with a high level of accuracy (i.e., over $90 \%$ recognition rate). In addition, we expected our classification procedure to be relatively non-sensitive to time synchronization issues. Spectrograms have been used extensively to extract information from such time-varying signals, and they show that the signals under study are nonstationary and vary in frequency content, magnitude, and background noise. The Discrete Wavelet Transform (DWT) provides an attractive alternative as it is better matched to mammal hearing, which could potentially lead to better classification performances.

Two main families of DWT decompositions are considered; orthogonal and non-orthogonal transforms. Orthogonal decomposition offer advantages in applications such as compression, where one aims at minimizing the amount of information transmitted. Two different bases are considered in our study; Symmlet-8 and Coiflet-3. Non-orthogonal transforms may have advantages in applications where the redundancy makes the information easier to extract $[2,4]$. The non-orthogonal transform considered was the undecimated A-trous implementation of the WT using a Morlet-type mother wavelet, as introduced in Shensa [4]. This undecimated transform has the additional advantage of being translation invariant.

\section{Feature Extraction Parameters Obtained using Wavelet Transforms}

Lemer et. al. showed in an earlier study that using energy quantities based on Daubechies orthonormal wavelets of order 6 improved classification performances for the specific underwater signals considered in their study [5]. Expanding on this idea, we use the average energy $E_{i}$ computed from the wavelet coefficients obtained at a given scale $i$ for scales 1 to 7 , and the complementary average energy contained in the low-pass operation. Thus, average wavelet-based quantities $E_{\mathrm{i}}$ for scale $i$ used as feature parameters are defined as:

$$
E_{i}=\frac{1}{2^{i}} \sum_{k} c_{i, k}^{2}, \quad i=1, \ldots, 7,
$$

where $c_{i, k}$ represents the $k^{\text {th }}$ wavelet coefficient obtained at scale $i$, and the summation operation is done over all wavelet coefficients available at a given scale. This study considers scales $i$ from 1 to 7 . A similar expression is used to derive the $2^{\text {nd }}$ set of coefficients from the low-pass operations in the same range of scales. As a result, a seven scale decomposition leads to a set of 14 real energy-type feature coefficients. Such a choice insures to keep the number of feature coefficients low, and avoids potential problems dealing with time-domain synchronization. Two orthonormal bases are used in the study and their performances compared; Coiflet- 3 and Symmlet -8 bases [1].

One of the main differences between orthogonal and non-orthogonal decompositions is the fact that the spectral partitioning (i.e., the frequency resolution) is fixed by the choice of a given orthogonal basis. However, in the non-orthogonal Atrous decomposition, the user may vary the spectral partitioning by changing the number of voices per octave, where voices can be viewed as sub-band filters, the roll-off parameter $\beta$, or the center frequency $\eta$ of the mother wavelet. The feature parameters chosen for the classification scheme are the set of average wavelet-based coefficients $A_{\mathrm{i}, \mathrm{j}}$ obtained at a given scale $i$ and voice $j$, where $A_{\mathrm{i}, j}$ is defined as:

$$
A_{i, j}=\frac{1}{2^{i}} \sum_{k}\left|c_{i, j, k}\right|^{2}, \quad i=1, \ldots, 7, \quad j=0, \ldots, m-1 .
$$

The parameter $c_{i, j, k}$ represents the $k^{\text {th }}$ wavelet coefficient obtained at scale $i$ and voice $j$, and the summation operation is conducted over the range of wavelets coefficients obtained at a given scale and voice. Thus, a seven scale decomposition using $m$ voices leads to $7 \mathrm{~m}$ input coefficients. Several configurations of the A-trous implementation are investigated in the study, using between 4 to 7 voices. Experiments were conducted using the roll-off parameter $\beta=.15$ and the center frequency $\eta=.85 \pi$ 
[1,3]. Results showed that the best overall classification results among the various implementations considered were obtained when using six voices per scale, as illustrated in Table I [1].

\section{Reduced-Rank AR Modeling}

AutoRegressive (AR) modeling is a timedomain technique used for modeling a set of data as the output of an all-pole LTI filter. Estimation of the filter coefficients may be carried out in a least squares sense by solving the Yule-Walker equations $[1,6]$. Degradations due to noise may be decreased by using a truncated inverse of the data matrix defined in the Yule Walker equations to solve for the AR coefficients. Such a truncated inverse is computed using the Singular Value Decomposition (SVD), and this approach has been used extensively in signal processing applications. The initial order chosen for the AR models was chosen to be 25 in our study, after visually inspecting the spectral densities of the data. The reduced-rank (i.e., the rank of the truncated inverse obtained using the SVD) was selected by detecting a visual gap in the singular values distributions of the data. Thus, the reducedrank varied between 2 and 17 for the data under study, where the smallest ranks was found for underwater earthquake and humpback data and the highest was found for sperm whale data [1].

Some of the underwater signals under study were buried in noise, and we attempted to decrease the effect due to wideband noise by applying an adaptive line enhancement (ALE) pre-processing step before computing the AR parameters. However, this pre-processing step was successful only on the four narrowband classes of signals, and produced mixed results on the other two. As a result, the overall classification rates did not improve when applying this pre-processing step, as illustrated in Table I.

\section{Classification Results}

Neural networks (NN) have been used extensively for classification purposes. They are useful in applications where the knowledge of the signals under study is not complete, as they can be trained by being shown training sets which have desired characteristics. Thus, an important part of the training procedure lies in selecting representative sets of the classes to be considered. A backpropagation neural network with two hidden layers is used in this study. The same data is used for training all different NN implementations. Each training class contains 87 sets of feature parameters. An average of 50 sets per testing class is used for the testing phase. Overall classification rates for the various Wavelet transforms considered at this point are presented in Table I, where each network configuration considered is described in terms of the number of inputs/number of PEs in the first hidden layer/number of PEs in the second hidden layer/number of output nodes.

Results show that the lowest overall classification rates are obtained when using orthogonal Wavelet transformations (around 78\%). Overall classification rates increase to $84 \%$ when using AR coefficients. Finally, results show that higher overall classification rates are obtained when using the non-orthogonal Wavelet transformation (between $93 \%$ and 96\%). Such results are to be expected as using multiple voices in the nonorthogonal transformation leads to a finer frequency decomposition of the signal information, which leads to a better match of the spectral information contained in the data under study.

\section{Conclusions}

This study compares the classification rates obtained when using Wavelet Transforms and AR modeling to select feature parameters as backpropagation NN inputs for classification purposes. Results show that the best overall classification rates are obtained when using the undecimated nonorthogonal A-trous implementation with multiple voices. These results are to be expected as the feature extraction scheme chosen for the Wavelet transforms can be viewed as an "energy-based" classifier, where the choice of the basis specifies the type of frequency partitioning used. The A-Trous implementation is well matched to the narrowband underwater data under study, as it leads to a finer frequency resolution than that obtained using orthogonal bases, which results in higher overall classification performances.

\section{References}

[1] R. Bennett, Classification of underwater signals, MSEE Thesis, Naval Postgraduate School, to be completed.

[2] O. Rioul and M. Vetterli, "Wavelets and Signal Processing," ASSP Magazine, pp. 14-38, Oct. 1991. 
[3] S. G. Mallat, "A Theory for Multiresolution Signal Decomposition: The Wavelet Decomposition," IEEE Trans. Patt. Anal. Machine Intell., Vol. 2, pp. 2091-2110, Dec. 1989.

[4] M. J. Shensa, "The Discrete Wavelet Transform: Wedding the A- Trous and Mallat Algorithms," IEEE Trans. on Signal Processing, Vol. 40, No. 10, pp. 2464-2478, Oct. 1992.

[5] A. Lemer, J.M. Nicolas, and P. Giancome,
"Identification Automatique de Bruits Impulsifs Sous-Marins," Proc. GRETSI, 1989.

[6] M.P. Fargues, R. Cristi, \& M.M. Vanderkamp," Modeling and Classification of Biological Signals Using Least-Squares Prony-SVD AR Models," Proc. $36^{\text {th }}$ Midwest Symposium Circuits and Systems, Detroit, MI, August 16-18, 1993, pp. 159-162.

Table I. Classification Rates

\begin{tabular}{|c|c|c|}
\hline Feature Extraction Method & Network Configuration & Classification Rate \\
\hline AR Coefficients (order 25) & $25 / 20 / 15 / 6$ & $84.7 \%$ \\
\hline ALE \& AR Coefs (order 25) & $25 / 20 / 15 / 6$ & $83.7 \%$ \\
\hline Wavelet (Symmlet 8) & $14 / 14 / 10 / 6$ & $78 \%$ \\
\hline Wavelet (Coiflet 3) & $14 / 14 / 10 / 6$ & $78.2 \%$ \\
\hline Wavelet A-Trous (4 voices) & $28 / 28 / 15 / 6$ & $96.4 \%$ \\
\hline Wavelet A-Trous (5 voices) & $35 / 20 / 15 / 6$ & $93.4 \%$ \\
\hline Wavelet A-Trous (6 voices) & $42 / 42 / 15 / 6$ & $96.7 \%$ \\
\hline Wavelet A-Trous (7 voices) & $49 / 49 / 15 / 6$ & $95.1 \%$ \\
\hline
\end{tabular}

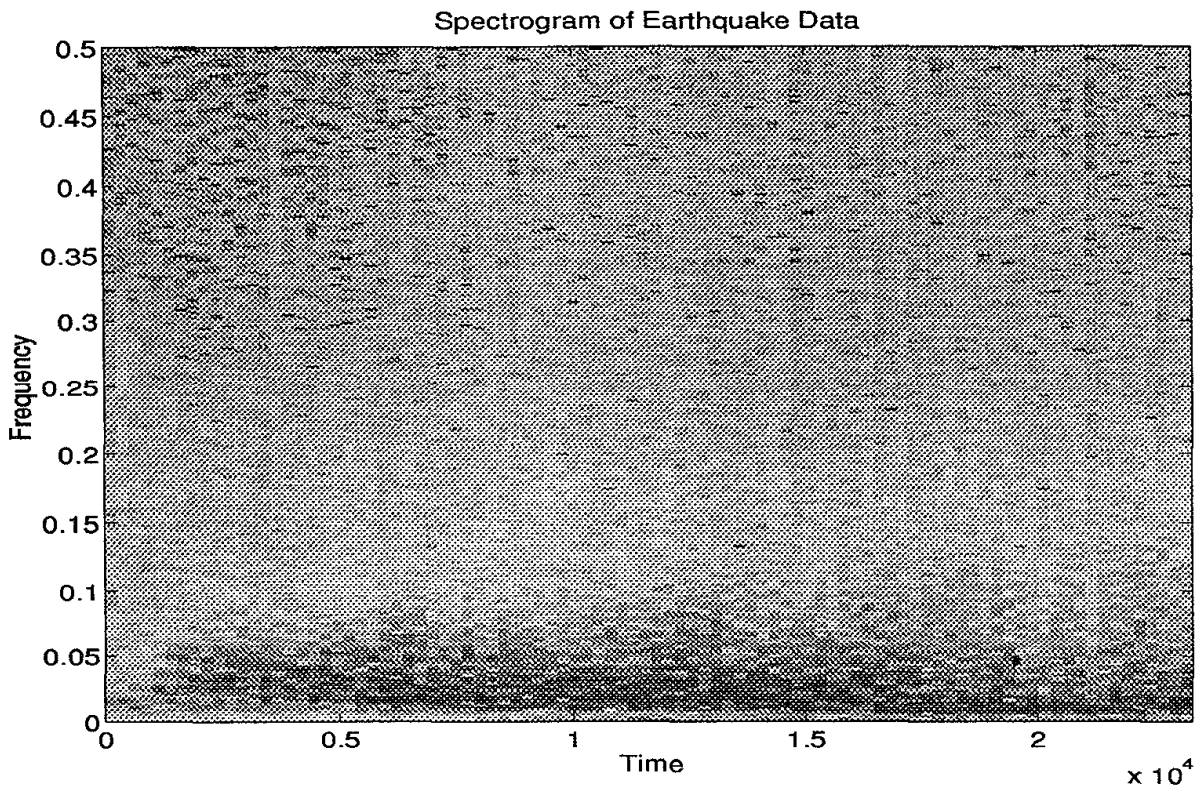

Figure 1. Spectrogram of underwater earthquake data; normalized frequency $\left(f_{s}=8 \mathrm{kHz}\right)$, normalized time (number of samples). 


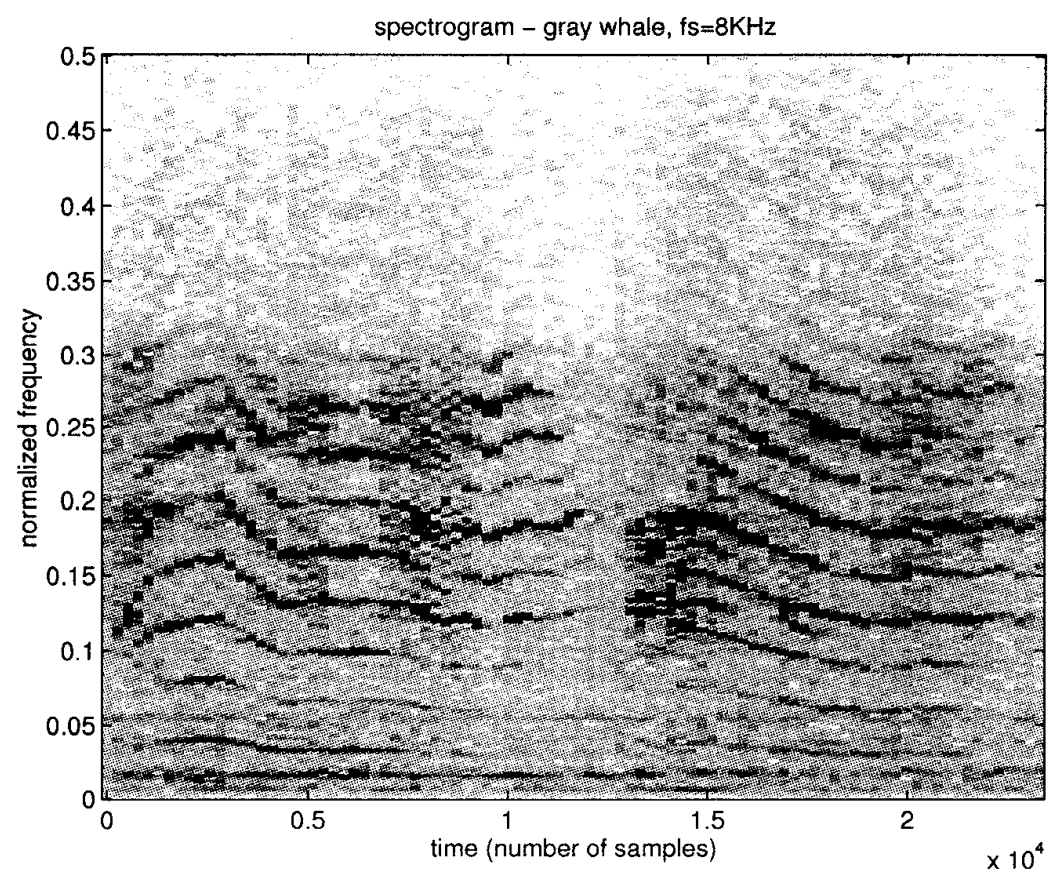

Figure 2. Spectrogram of gray whale data; normalized frequency $\left(f_{s}=8 \mathrm{kHz}\right)$, normalized time (number of samples).

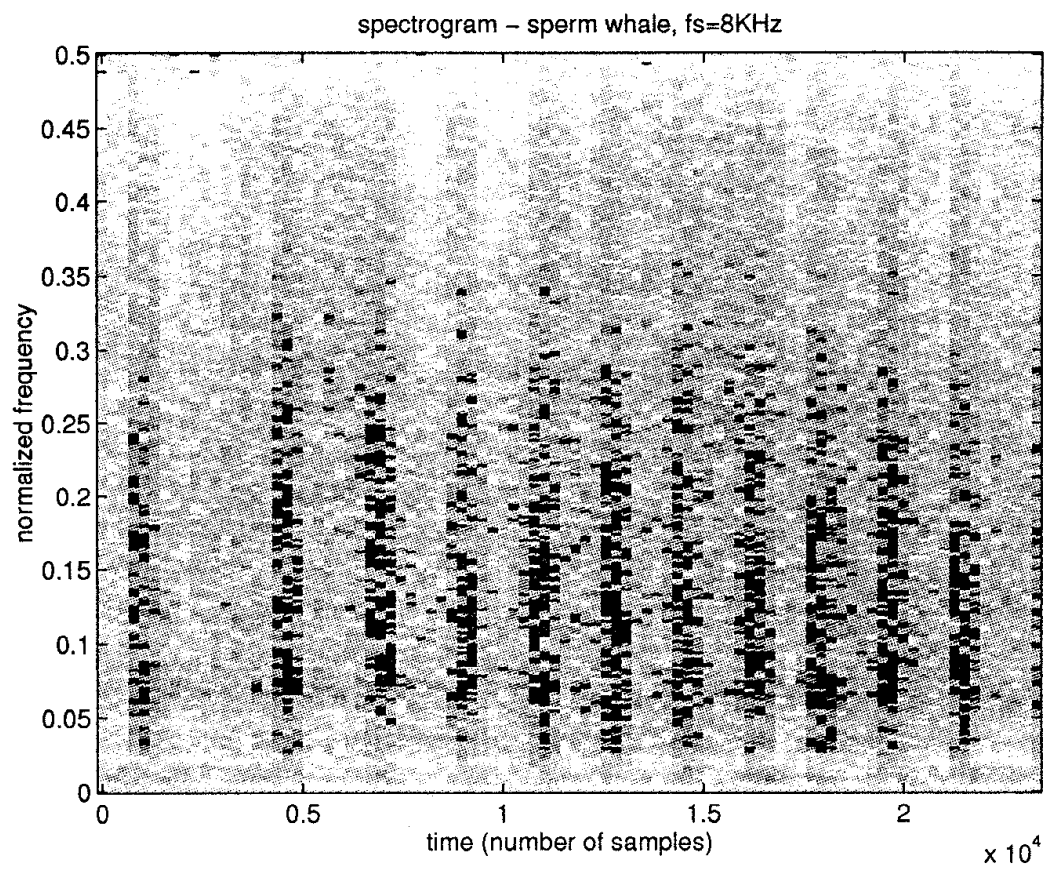

Figure 3. Spectrogram of sperm whale data; normalized frequency $\left(f_{s}=8 \mathrm{kHz}\right)$, normalized time (number of samples). 